\title{
The REDTOP project: Rare Eta Decays with a TPC for Optical Photons
}

\author{
Corrado Gatto \\ Istituto Nazionale di Fisica Nucleare - Sezione di Napoli \\ E-mail: gatto@na.infn.it \\ Brenda Fabela Enriquez ${ }^{* \dagger}$ \\ Universidad Autónoma de Zacatecas \\ E-mail: brenda.fabela@fisica.uaz.edu.mx
}

\section{Maria Isabel Pedraza Morales}

Benemérita Universidad Autónoma de Puebla

E-mail: mpedraza@cern.ch

\begin{abstract}
The $\eta / \eta^{\prime}$ mesons are almost unique in the particle universe since they are Goldstone boson and the dynamics of their decays are strongly constrained. Because the $\eta$ and $\eta^{\prime}$ have all zero quantum numbers, decays that violate conservation laws can occur without interfering with a corresponding current. The integrated eta meson samples collected in earlier experiments have been less than $\sim 10^{8}$ events, limiting considerably the search for such rare decays. The $\eta^{\prime}$ sample is even more scarce. A new experiment REDTOP, is being proposed at the proton booster of Fermilab with the intent of collecting more than $10^{12}$ triggers/year for studies of rare $\eta$ decays. Such statistics are sufficient for investigating several symmetry violations, and for searching for new particles beyond the Standard Model. The physics program, the accelerator systems and the detector for REDTOP were presented in this conference.
\end{abstract}

38th International Conference on High Energy Physics 3-10 August 2016

Chicago, USA

\footnotetext{
* Speaker.

${ }^{\dagger}$ for the REDTOP collaboration.
} 


\section{Introduction}

REDTOP (Rare Eta Decays with a TPC for Optical Photons) is a new project being proposed at Fermilab. It belongs to the high intensity class of experiments as it aims at detecting small variations from the Standard Model by looking at a large number of events produced with very intense beams.

This experiment aims at producing about $2 \times 10^{12} \eta$ mesons in one year of running by using a $1.9 \mathrm{GeV}$ continous proton beam impinging on a target composed by 10 foils of beryllium. Those characteristics are making REDTOP essentially an $\eta$ factory, capable of studing the decay products of the $\eta$ that are either not expected or suppressed at the $10^{-11}$ level. The detector for this experiment requires to be blind to protons and slow charged pions, all copiously produced in the targets along with the $\eta$ mesons. Such background has inherently slow speed and it will not be detected by an experimental apparatus based on production of Cherenkov light. On the other hand, such detector woud be sensitive to leptons and fast pions which are present in the final state of most rare $\eta$ decays.

\section{Motivation}

The $\eta$ meson is associated with a wide variety of interesting nuclear and particle physics. It is a Goldstone boson, so the symmetry constrains its QCD dynamics. All its additive quantum numbers are zero: that includes the spin, isospin, electric charge, strangeness and other quark-flavor numbers, lepton number and baryon number and therefore its decays are not influenced by a current changing the flavor (as in $K$ decays).

The $\eta$ meson has G-parity +1 which mixes with the $\pi^{0}$ meson which has G-parity -1 , inducing potentially large charge-symmetry breaking. The $\eta$ is an eigenstate of the charge conjugation operator $\mathbf{C}$, and of $\mathbf{C P}: I^{G} J^{P C}=0^{+} 0^{-+}$, which is very rare in nature and it makes possible to use it for tests of $\mathbf{C , ~} \mathbf{C P}$ and even $\mathbf{C P T}$ invariance in flavor-non-changing interactions in rare $\eta$ decays [1].

All its strong decays are forbidden in lowest order by $\mathbf{P}$ and $\mathbf{C P}$ invariance, $\mathbf{G}$-parity conservation and isospin and charge symmetry invariance as well as all its electromagnetic decays are forbidden in lowest order by $\mathbf{C}$ invariance and angular momentum conservation. Hence, the $\eta$ meson is a very narrow state $\left(\Gamma_{\eta}=1.3 \mathrm{keV}\right)$ and the contributions from higher order processes are enhanced by a factor of $\sim 10^{5}$. Finally, the $\eta$ decays are flavor conserving reactions, thus the decays are free of Standard Model backgrounds for new physics searches.

A summary of the present and future $\eta$ meson samples is given in Table 1 .

\section{The physics program}

The physics program that REDTOP intends to carry can be divided in three main areas: (1) symmetry and conservation violations, (2) searches for new particles and forces and (3) low and intermediate enery QCD studies. 
Table 1: Present and future $\eta$ samples.

\begin{tabular}{ccc}
\hline \hline Experiment & Technique & Total $\eta$ \\
\hline CB at AGS & $\pi^{-} p \rightarrow \eta n$ & $10^{7}$ \\
CB at MAMI-B & $\gamma p \rightarrow \eta p$ & $2 \times 10^{7}$ \\
CB at MAMI-C & $\gamma p \rightarrow \eta p$ & $6 \times 10^{7}$ \\
KLOE & $e^{+} e^{-} \rightarrow \phi(1020) \rightarrow \eta \gamma$ & $5 \times 10^{7}$ \\
WASA at COSY & $p p \rightarrow \eta p p, p d \rightarrow \eta^{3} H e$ & $>10^{8}, 3 \times 10^{7}$ \\
CB at MAMI & $\gamma p \rightarrow \eta p$ & $3 \times 10^{8}$ \\
GlueX at JLab (proposed) & $\gamma p \rightarrow \eta p \rightarrow$ neutrals & $4.5 \times 10^{7} / y r$ \\
REDTOP at FNAL (proposing) & $p p \rightarrow \eta p p, p n \rightarrow \eta p p$ & $2 \times 10^{12}$ \\
\hline
\end{tabular}

\section{Symmetry and conservation violations}

- CP Violation (Type I: P And T OdD, C EVEn): The parity of the final state is reversed, but its charge conjugation is not. This situation occurs when the $\mathbf{P}=-1$, then, the $\eta$ meson decays into an even number of pions. The final state of interest for this study is:

$$
\eta \rightarrow 4 \pi^{0} \rightarrow 8 \gamma
$$

- CP Violation (Type II: C And T OdD, P EVEn): The charge of the final state is reversed, but its parity is not. This occurs for the following class of decays:

$$
\eta \rightarrow \pi^{0} l^{+} l^{-} \quad \eta \rightarrow 3 \gamma
$$

The decay $\eta \rightarrow \pi^{0} l^{+} l^{-}$violates $\mathbf{C}$ and $\mathbf{C P}$ if it occurs via a single photon intermediate state.

- CP Violation via the polarization of the muons: The CP invariance does not allow the final state muons in the decay

$$
\eta \rightarrow \mu^{+} \mu^{-}
$$

to be logitudinally polarized. Any polarization requires a $\mathbf{C P}$ violating lepton-quark current. An immediate implication of observing a non-zero longitudinal polarization of the muons is the existence of an extra Higgs boson. If that is the case, the muon polarization would be as high as $10^{-2}$.

- CP and C Violation with Dalitz plot studies: The $\mathbf{C P}$ invariance requieres that in the decay

$$
\eta \rightarrow \pi^{+} \pi^{-} \pi^{0}
$$

the dynamics of the charged pions are totally symmetric. Consequently, the Dalitz plot of a decay should show no signs of asymmetries. On the contrary, any mirror-asymmetry in the Dalitz plot would be an indication of the $\mathbf{C P}$ and $\mathbf{C}$ violation. 
- T Violation Via the polarization of the muOns: The $\mathbf{T}$ invariance requires a null transverse polarization of the muons in the decay

$$
\eta \rightarrow \pi^{0} \mu^{+} \mu-\quad \text { and } \quad \eta \rightarrow \gamma \mu^{+} \mu^{-}
$$

Any observed polarization of the muons is a direct violation of $\mathbf{T}$ invariance.

- CPT Violation: The CPT invariance can be probed with the following two processes:

$$
\eta \rightarrow \pi^{+} \mu^{-} \overline{v_{\mu}} \quad \text { vs } \quad \eta \rightarrow \pi^{-} \mu^{+} v_{\mu}
$$

If CPT holds, then we expect that the transverse polarization of the $\mu^{-}$and that of the $\mu^{+}$ be reversed. Namely, $P T\left(\mu^{+}\right)=-P T\left(\mu^{-}\right)$. As an aside note, the $\eta \rightarrow \pi \mu \nu$ has not been observed yet.

- CPT Violation with nOn-hermitian lagrangians: The CPT invariance and hermiticity of the lagrangian implies that in the following processes:

$$
\eta \rightarrow \gamma \gamma \quad \text { and } \quad \pi^{0} \rightarrow \gamma \gamma
$$

the photons have null circular polarization. There are some indications that the lagrangians for physical processes do not have necessarily to be Hermitian [2]. The observation of a non-null circular polarization of the photons is an evidence of CPT violation and of nonhermiticity of the lagrangian as well.

- LEPTON flavor ViOlation: Violation of the leptonic number will be observed through the following process:

$$
\eta \rightarrow \mu^{+} e^{-}+c . c . \quad \text { and } \quad \eta \rightarrow \pi^{0} \mu^{+} e^{-}+\text {c.c. }
$$

Current PDG limits are $<5.7 \times 10^{-13}, \mathrm{CL}=90 \%$ (from the direct muon decay $\mu^{-} \rightarrow e^{-} \gamma$ ).

- Double lepton flavor violation: Double violation of the leptonic number will be observed through the following process:

$$
\eta \rightarrow \mu^{+} \mu^{+} e^{-} e^{-}+c . c .
$$

This is expected a very rare process to occur. Nonetheless, if it occurs within the sensitivity of the experiment, it will be easily detected as it carries a very unique signature.

\section{Searches for new particles and forces}

- SEARCh FOR TRUe MUONIUM: True muonium is a bound state of a $\mu^{+} \mu^{-}$pair with a well defined mass of about $2 m_{\mu}$ [3]. The processes involved are:

$$
\left.\eta \rightarrow \gamma\left(\mu^{+} \mu^{-}\right)\right|_{2 m_{\mu}} \rightarrow \gamma e^{+} e^{-}
$$

This state has not been observed yet. Detection of true muonium would be an important discovery and would represent a further important test of QED. 
- SEARChes For DARK PHOtons: The dark (or heavy) photon $A^{\prime}$ is a $U(1)$ vector boson, mediator of a possible new force. From the astrophysical observations of several anomalies, the mass of such particle is expected to be in the $\mathrm{GeV}$-scale. If it is below the mass of the $\eta$ meson it can be observed via the process:

$$
\eta \rightarrow \gamma A^{\prime} \rightarrow \gamma+l^{+} l^{-}
$$

The $\eta$ and the $A^{\prime}$ mass constraints will help to reject the background.

- SEARCHES FOR NEW BARYONIC FORCES: From the experimental point of view, this search is similar to the dark photon case, but without the mass constraint on the resonance. If the vector boson $B$ mediating that force is below the mass of the $\eta$ meson it can be observed via

$$
\begin{array}{ccc}
\eta \rightarrow \gamma B & \text { with } & B \rightarrow \gamma \pi^{0} \\
\eta \rightarrow \gamma B & \text { with } & B \rightarrow \gamma l^{+} l^{-}
\end{array}
$$

New forces in the $\mathrm{MeV}-\mathrm{GeV}$ range associated with dark matter are postulated by several models [4], [5], [6], [7], [8], [9].

- SEARCHES FOR LEPTOQUARKS: Leptoquarks are particles mediating a new interaction between quarks and leptons of the same generation. They are color-triplet bosons that carry both lepton and baryon numbers. Observing any discrepancy between the rates measured and predicted by the Standard Model in the processes

$$
\eta \rightarrow \mu^{+} \mu^{-} \quad \text { and } \quad \eta \rightarrow e^{+} e^{-}
$$

would be an indication of additional contributions from new diagrams and an indication of the existence of such particles..

\section{Low and intermediate energy studies}

More, high precision, studies that could be performed using the $\eta$ meson sample collected by REDTOP include:

- Test of nuclear models

- Chiral perturbation theory

- Non-perturbative QCD

- Isospin breaking due to the $u-d$ quark mass difference

- Octet-singlet mixing angle

- $\pi \pi$ interactions

- Electromagnetic transition form-factors 


\section{The experimental apparatus}

\subsection{Beam and target}

REDTOP will produce the $\eta$ mesons via the hadronic production from inelastic scattering of protons on beryllium targets. The hadronic production cross section for the $\eta$ is quite large (several tens of $\mathrm{mb}$ ) in the $1.9-2.1 \mathrm{GeV}$ energy range. At such beam energies, the $\eta$ mesons are produced mostly resonantly inside the nuclear matter of the target and their kinematics is mostly unaffected from variations of the energy of the proton beam up to about $200 \mathrm{MeV}$.

The experiment will use a Continous Wave $(\mathrm{CW})$ proton beam with an energy of $1.9 \mathrm{GeV}$ and an intensity of $10^{11}$ protons / sec. The target systems is composed of ten round foils of beryllium, each of about $1 / 3 \mathrm{~mm}$ thickness and $1 \mathrm{~cm}$ diameter. The target systems will be held inside a beam pipe made of either carbon-fiber or beryllium. The target is sparsified with multiple elements along the beam axis, helping to reduce the pile-up since the signal event and the background event will likely occur in different elements.

Simulations conducted with the GenieHad-Urqmd and GenieHad-Inclxx framework [10] indicate that a beam with these characteristics has a probability of $0.5 \%$ to interact inelastically with the target systems. The probability that one of such events will produce an $\eta$ meson is about $0.4 \%$. Therefore, this beam will produce about $2 \times 10^{5} \eta / \mathrm{sec}$ or $2 \times 10^{12} \eta /$ year.

The total beam power necessary is less that $30 \mathrm{~W}$. Each of the ten foils comprising REDTOP target systems will absorb, on average, about $15 \mathrm{~mW}$ of power, which can be easily dissipated without a dedicated cooling system.

\subsection{The accelerator complex}

An acceleration and beam delivery systems that can be used for REDTOP is currently being investigated. At present the beam facilities at Fermilab do not comprehend a beam within REDTOP's requirements. However, the Booster system is capable of delivering protons at a fixed energy of $8 \mathrm{GeV}$ with an intensity exceeding that required by REDTOP. The acceleration and beam delivery system for REDTOP are under deep investigation by Fermilab's Accelerator Division. The centerpiece of this design will be the Delivery Ring, a refurbishing of a storage ring used during the production of antiprotons for the Tevatron. The current design is based on sending $8 \mathrm{GeV}$ beam transferred from the Booster to the Delivery Ring. Then, the protons are decelerated to the required energy. Finally, the protons are slowly extracted to the experimental area. Alternative scenarios are also being investigated.

According to the present design, a single proton pulse from the Booster can contain up to $4 \times 10^{12}$ protons. Such pulse, after a deceleration to approximately $2 \mathrm{GeV}$ and a slow extraction to the REDTOP target over approximately 40 seconds, would be able to produce $\eta$ mesons at a rate sufficient for REDTOP's physics program. A schematic of REDTOP accelerator complex is shown in Fig. 1. 


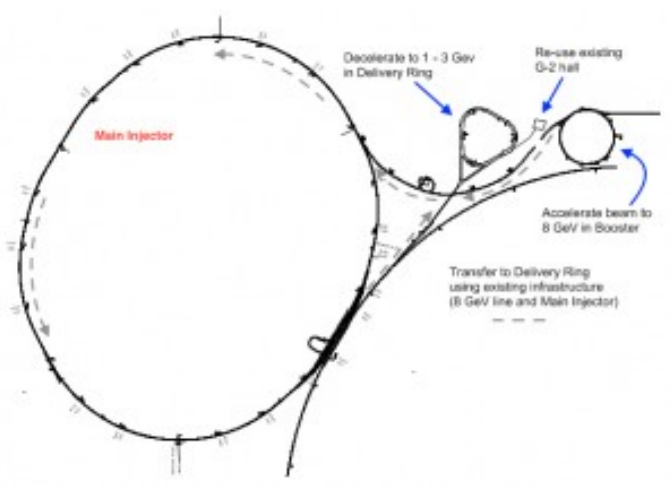

Figure 1: Schematics of the REDTOP accelerator complex.

\subsection{The detector}

The main challenge for the REDTOP experiment is represented by the rejection of the background generated by the beam in the target systems, since, at the energy where the experiment will run, the ratio of $\eta$ production to inelastic proton scattering is about 1:200. In order to make the signal-to-noise ratio more favorable, the apparatus will use the Cherenkov process to detect the fast particles (essentially leptons and a fraction of pions) in the events of interest . By tuning appropriately the refractive index of the radiators, the background events, with mostly hadrons in the final states, will be largely invisible to the detector.

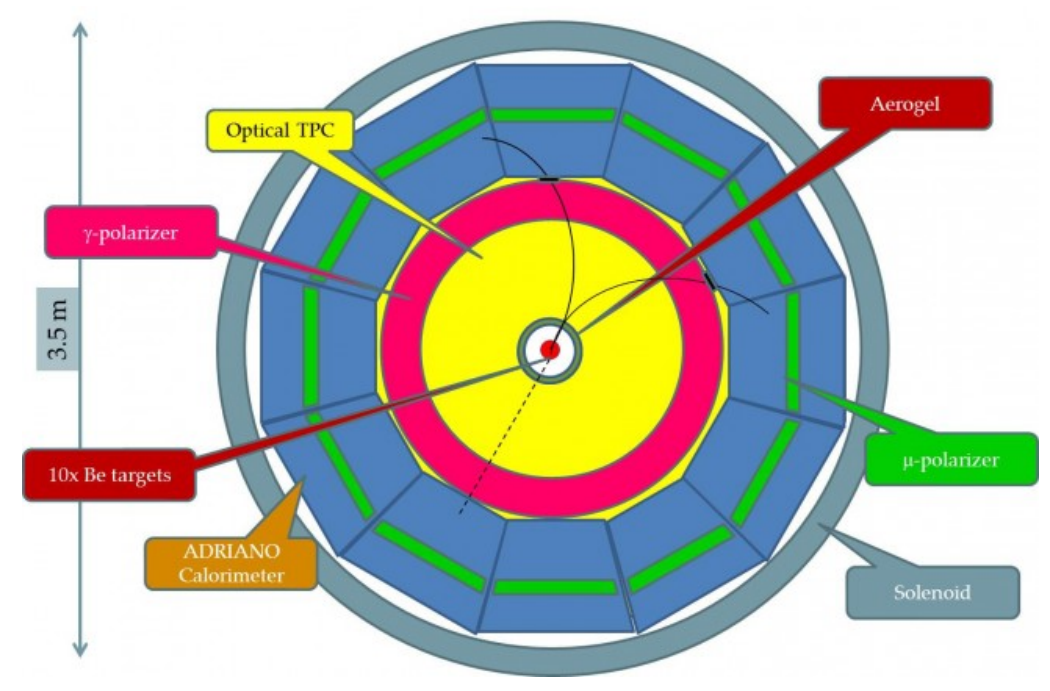

Figure 2: Schematics of the REDTOP detector.

The REDTOP detector is composed by several subsystems: the Optical Time Projection Chamber (OTPC), the ADRIANO Dual-Readout Calorimeter, the Muon Polarimeter and an (optional) Photon Polarimeter. 


\section{The Optical Time Projection Chamber}

The principles of operation of the Optical Time Projection Chamber (OTPC) are similar to a conventional TPC, namely, it will measure the momentum and position in space of a charged track by using a deflecting solenoidal field. However, instead of using the ionization process to detect those tracks, it will use the Cherenkov effect. The momentum of the particle will be reconstructed by the photons emitted inside the volume of the OTPC from gas and aerogel radiators and detected by an array of photo-sensors surrounding the chamber.

Two Cherenkov radiators are present in the OTPC:

- A double aerogel cylinder, about $3 \mathrm{~cm}$ thick at the inner wall, being supported by the beam pipe. The innermost aerogel has $n_{D}=1.22$, while the outermost has $n_{D}=1.3$.

- A low pressure nitrogen gas filling the bulk of the volume of the OTPC. The pressure is adjusted in order to have $n_{D}=1.000145$.

Muons with momentum larger than $160 \mathrm{MeV}$ will radiate only in the aerogel. The radius, the center and the skewness of the ring will help to determine their velocity. The dual refractive systems will help in discriminating the pions from the muons. The electrons and positrons will radiate in aerogel as well as in the gas and they will produce a ring with a radius mostly independent from their velocity and much larger than that of the muons. The momentum of them will be extracted by the pattern of photons collected by the photosensors. The solenoid used by REDTOP will have a magnetic field of $0.6 \mathrm{~T}$.

The advantage of an OTPC is that it will be sensitive only to those fast particles (leptons and pions) that are generated in the decays of the $\eta$ meson of interest. Hadrons and slower pions will be under the Cherenkov threshold and, consequently, will not contribute to the recorded hits.

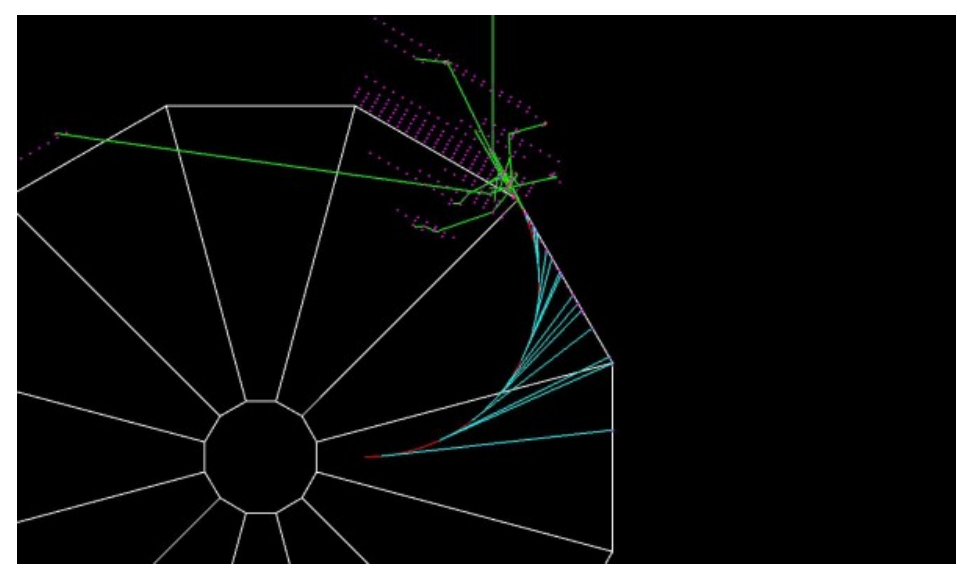

Figure 3: Representation of a $100 \mathrm{MeV}$ electron (red track) traveling through the gas in a $0.6 \mathrm{~T}$ magnetic field. Several Cherenkov protons (cyan tracks) are generated and detected by the optical sensors surrounding the gas. 


\section{The ADRIANO Dual-Readout Calorimeter}

A multiple-readout calorimeter is being chosen for REDTOP beacuse of its particle identification capabilities. The latter are very important in order to disentangle the signal produced by leptons and photons from the very abundant hadron background (mostly neutrons). The implementation of the multiple-readout calorimetric technique chosed for REDTOP is named ADRIANO (A Dual-Readout Integrally Active Non-segmented Option) [11]. It is based on the two simultaneous measurements of the energy deposited by a hadronic or electromagnetic shower into two media with different properties. A third independent measurement (based on the time structure of the electronic signals) will help to separate the neutron component of the shower. The first medium is usually a plastic scintillator. Then, any charged particle depositing energy in that medium will produce scintillation and will make a signal in the corresponding electronics.

The second medium is usually a heavy glass with high refractive index and high density. This medium will only be sensitive to charged particles with large velocity, such that light is emitted via the Cherenkov effect and only the charged electromagnetic component of the shower or photons producing pais will produce a signal. The high density of the medium will make it an ideal integrally active absorber for all particles impinging on the detector.

Summing the Cherenkov (C) and the scintillation (S) signal it could be obtained the total energy of the detected particle. Particle identification is achieved by comparing the scintillation (S) vs. the Cherenkov (C) signals obtained in the two separated optical media. The neutron component $\mathrm{N}$ has a different time structure which can be identified by instrumenting the scintillating medium with FADC's.

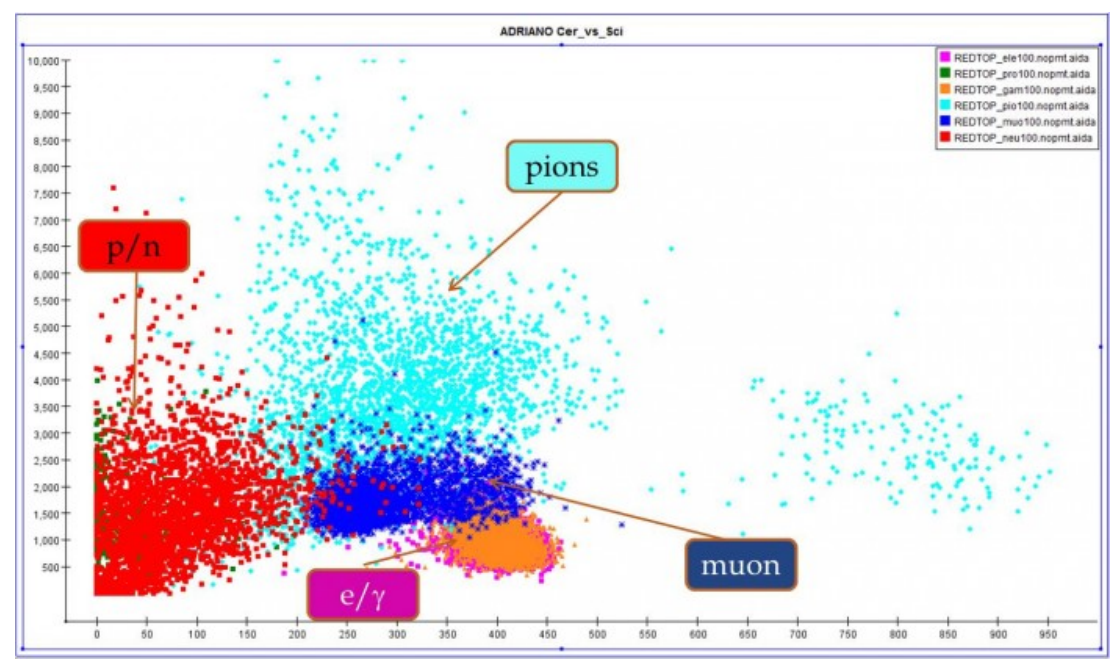

Figure 4: Particle identification from the comparison between the Scintillation vs. Cherenkov signals in the ADRIANO calorimeter for simulated particles with $E_{k i n}=100 \mathrm{MeV}$.

\section{The Muon and Photon Polarimeter}

The muon and photon polarimeters are made from an array of plastic scintillators inserted 


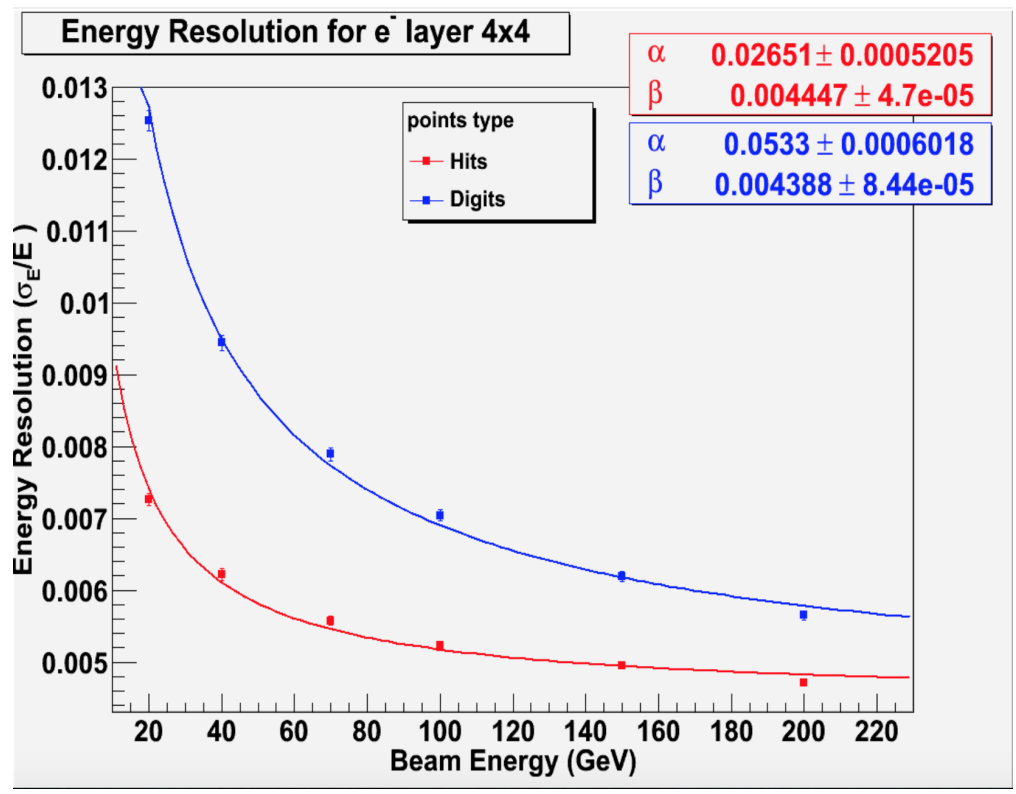

Figure 5: Energy resolution of the ADRIANO calorimeter simulated with ilcroot.

between the inner and outer shells of the ADRIANO calorimeter and a $5 \mathrm{~mm}$ thick scintillating plate inserted inside the volume of the OTPC respectively. They will count the number of electrons and positrons emitted when a muon is stopped inside the ADRIANO calorimeter or when a photon is converted into an $e^{+} e^{-}$pair. The asymmetry in the counting performed inside the solenoidal magnetic field will provide an estimate of the polarization of the decaying particle. At present, the photon polarimeter is optional, as it is only required by the studies on CPT violation.

\section{The running plan}

The experiment is initially planned to run at one energy, but its physics program could be extended into two more (optional) runs:

- RUN I. The REDTOP experiment will be in all respects an $\eta$-factory. The beam energy will be approximately $1.9 \mathrm{GeV}$. Approximately, $10^{12} \eta$ mesons will be produced.

- RUN II-OPTIONAL. The detector will be refurbished (by replacing the aerogel) for the use of a $3.5 \mathrm{GeV}$ proton beam, which is above the $\eta^{\prime}$ production threshold, making REDTOP an $\eta^{\prime}$-factory. Approximately, $10^{10} \eta^{\prime}$ mesons will be produced.

- RUn III-OPTIOnAL. The main physics topic will be the study of the CP violating process $K^{+} \rightarrow \pi^{+} v v$. The kaon beam will be generated as a secondary from a primary of $8 \mathrm{GeV}$ proton beam. The O-TPC and the target need to be replaced. The goal is to reconstruct several hundred $K^{+} \rightarrow \pi^{+} v v$ decays. 


\section{Expectations and conclusions}

The $\eta / \eta^{\prime}$ mesons are an excellent laboratory for searching for physics Beyond the Standard Model, provided that an experiment could be designed to produce an excess of $10^{12}$ of such particles. REDTOP is being designed to do exactly for this, with several novel detector techniques which are highly sensitive to processes from new physics, but mostly insensitive to background from old physics.

\section{References}

[1] B. M. K. Nefkens, What is so special about eta-meson physics in Mesons and Light Nuclei'95, Springer, Vienna 1995.

[2] L. B. Okun, C, P, T are Broken. Why Not CPT? [hep-ph/0210052].

[3] S. J. Brodsky and R. F. Lebed, Production of the smallest QED atom: true muonium $(\mu+\mu-)$, Phys. Rev. Lett., 102 (21), 213401.

[4] N. Arkani-Hamed, D. P. Finkbeiner, T. R. Slatyer and N. Weiner, A theory of dark matter, Phys. Rev. $D, 79$ (1), 015014.

[5] M. Pospelov and, A. Ritz, Astrophysical signatures of secluded dark matter, Phys. Lett. B, 671 (3), 391-397.

[6] M. Kaplinghat, S. Tulin and H. B. Yu, Self-interacting dark matter benchmarks, arXiv:1308.0618 [hep-ph].

[7] J. L. Feng, M. Kaplinghat, H. Tu and H. B. Yu, Hidden charged dark matter, JCAP, 07 (2009) 004, [arXiv:0905.3039].

[8] A. Loeb and N. Weiner, Cores in dwarf galaxies from dark matter with a Yukawa potential. Phys. Rev. Lett., 106 (17), 171302.

[9] T. Lin, H. B. Yu and K. M. Zurek, Symmetric and asymmetric light dark matter, Phys. Rev. D, 85 (6), 063503.

[10] http://redtop.fnal.gov/the-geniehadevent-generation-framework/

[11] C. Gatto, V. Di Benedetto, E. Hahn and A. Mazzacane, Status of Dual-readout R\&D for a linear collider in T1015 Collaboration, arXiv:1603.00909 [physics.ins-det].

[12] N. Akchurin, F. Bedeschi, A. Cardini, M. Cascella, F. Cei, D. De Pedis and P. Genova, Dual-readout Calorimetry, arXiv:1307.5538 [physics.ins-det]. 